\title{
Multicentric epitheloid haemangioendothelioma of bone
}

S T Hlongwane, MB ChB
H B Louw, MBChB, MFamMed
G Dekker, MB ChB, MFamMed
K M Naidu, MBChB, MMedRad
M Pienaar, BSC, MB ChB
S Andronikou, MB BCh, FCRad, FRCR, PhD
Department of Radiology, Stellenbosch University and Tygerberg Hospital

\section{Abstract}

Multicentric epitheloid haemangioendothelioma (EHE) of bone is a rare primary bone tumour which may present at any age. The diagnosis is suspected on radiographs and the differential diagnosis includes metastatic disease, epitheloid angiosarcoma and EHE. Confirmation is only by means of biopsy and histological examination.

Multiple lesions and metastases are confirmed or excluded by further imaging such as radiographs, whole-body nuclear bone scan and CT scan of the chest. When positive, these findings change the prognosis and approach to treatment.

In our case an elderly man presented with a pathological fracture of the right tibia as a complication of EHE. Diagnosis was suspected on radiographs and confirmed on histology. Whole-body bone scintigraphy and CT scan of the chest were performed, which confirmed multiple bone lesions and metastases to the lungs.

\section{Introduction}

Haemangioendothelioma is the term used to describe vascular neoplasms presenting with histological features and clinical behaviour intermediate between benign well-differentiated haemangiomas and anaplastic angiosarcomas. ${ }^{1}$

They can occur in the liver, skin, soft tissue, heart, central nervous system and many other locations. ${ }^{1-3}$ Less frequently they are found in the bone, accounting for less than $0.5-1 \%$ of all primary bone neoplasms. ${ }^{1,2}$ The tumour is mainly located in the long tubular bones of the lower extremity and the pelvis, less frequently in the vertebral column, upper extremity, and flat bones. ${ }^{2}$ Cases have also been described involving the skull. $^{1}$

All age/sex groups can be affected by the tumour, but there is predilection for the 4 th - 6 th decade. ${ }^{1,2}$

\section{Case report}

A male patient, age 79 years, presented with a pathological fracture of the right tibia. Radiographs showed multiple lytic lesions of the right femur and tibia. The lesions were well circumscribed with no associated sclerosis or soft tissue mass (Figs. 1a - c). Pelvic, lumbosacral and chest $\mathrm{X}$-rays revealed additional lytic bone lesions in the pelvis (Fig. 1d). An open reduction and internal fixation together with open biopsy were performed.
The initial diagnosis was that of metastatic disease. Histological analysis demonstrated epitheloid haemangioendothelioma (EHE) and no features of angiosarcoma. The patient was referred to a tertiary hospital for further management.

A CT scan of the lung revealed lung metastases and diffuse alveolar airspace disease in keeping with infection (Fig. 2a). On bone windows a lytic lesion was detected in the third lumbar vertebra. Whole-body scintigraphy using TcMDP $905 \mathrm{MBq}$ demonstrated osteoblastic lesions in both femurs and the third lumbar vertebra (Fig. 2b).

The patient unfortunately died due to Gram-negative sepsis.

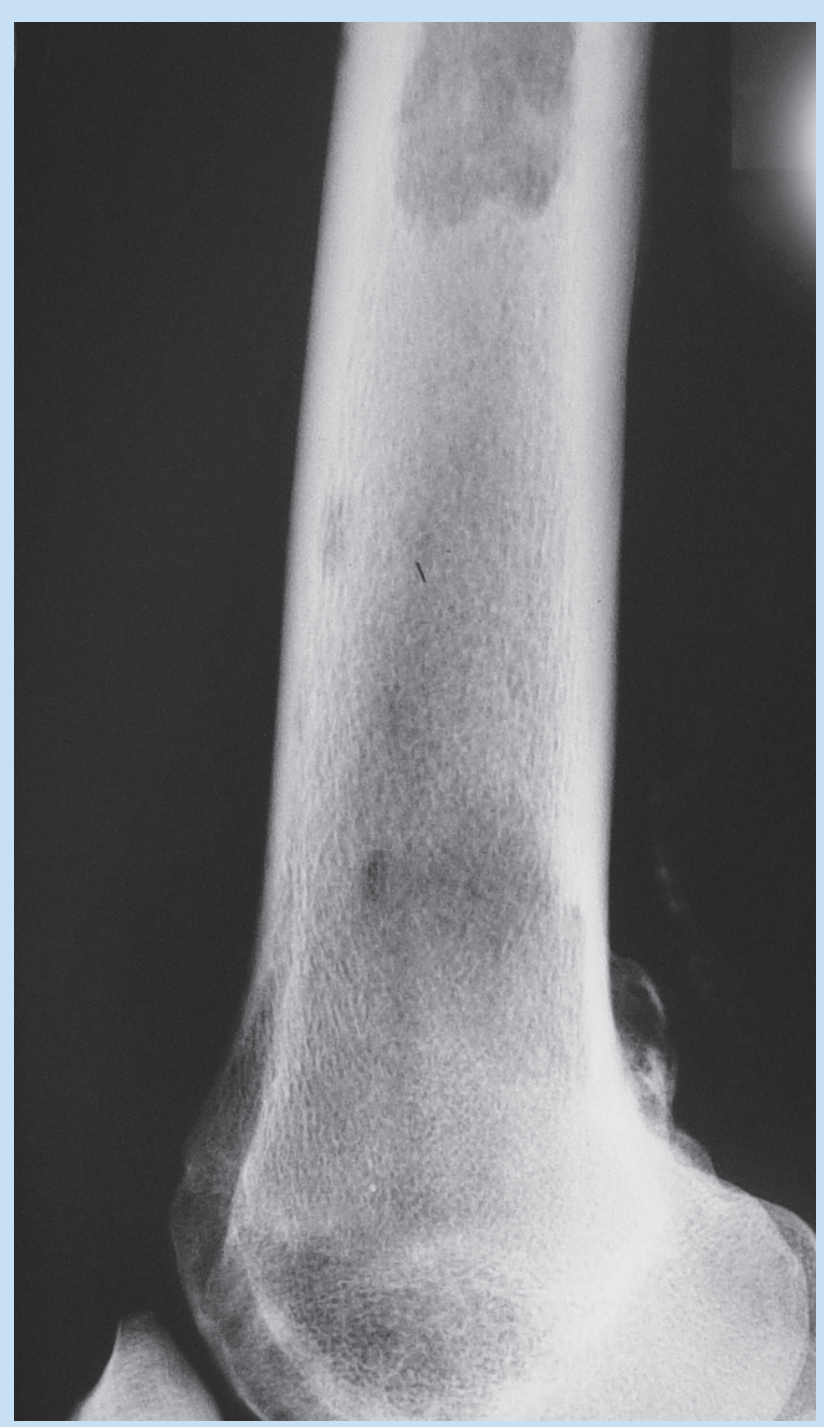

Figs. $1 a-d$. Conventional $X$-rays of the right knee, femur and pelvis. Multiple lytic lesions, some well circumscribed and others permeative, in the right tibia, femur and pelvis. 


\section{CASE REPORT}

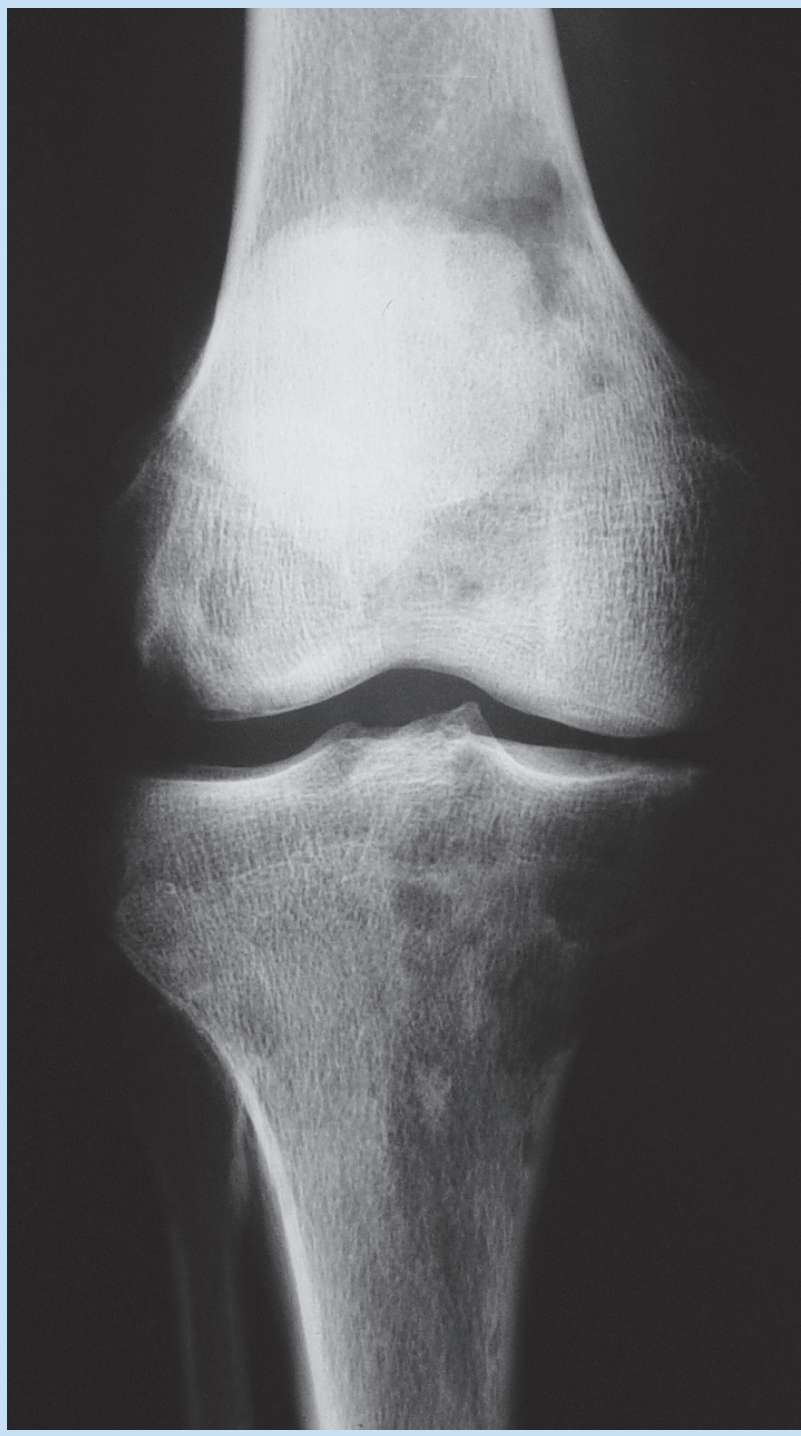

Fig. $1 b$.

\section{Discussion}

There are two forms of haemangioendothelioma, viz. epitheloid type and spindle-cell type. ${ }^{1}$ The epitheloid type is a well-differentiated endothelial vascular neoplasm, with an epitheloid appearance to the endothelial cells and a tendency to be multifocal. The spindle cell type is a rare tumour of the endothelial cells that resembles cavernous haemangioma. EHE of bone is a low-grade malignant vascular tumour with a relatively benign course in contrast to the poorly differentiated angiosarcoma. There is varying opinion about its malignant potential, prognosis, and clinical course. ${ }^{2,4}$ For some authors ${ }^{1,2}$ the most important indicator is the histological grade of the tumour.

The tumor is graded I - III according to the amount of cellular atypia present, with grade III being the most malignant.

With occurrence of metastases or lesions in other organs the prognosis is poor. ${ }^{1,2}$ Metastatic disease occurs most often to the lung.

In up to $55 \%$ of cases the disease is multicentric and often multicompartmental..$^{1,2}$ Multicentric appearance is either metachronous or synchronous. ${ }^{2}$

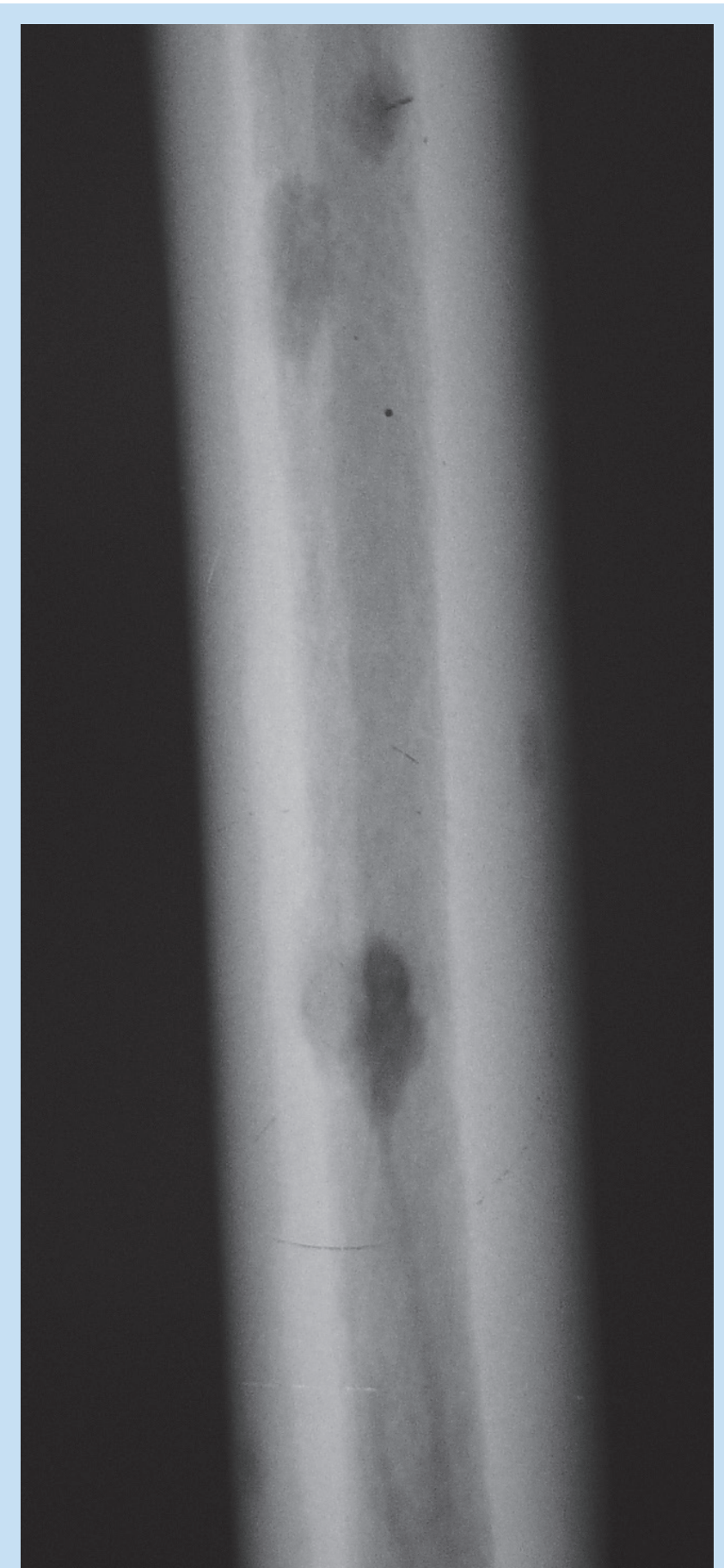

Fig. 1c.

The rarity of this tumour and the uncharacteristic clinical symptoms of pain and sometimes swelling may make its clinical detection difficult. Plain film radiography is the best diagnostic procedure for the initial imaging of the tumour. ${ }^{2,3}$

As in our patient, the findings on plain film radiography are multiple small osteolytic lesions which may have a more permeative character. $^{2}$ Sclerosis and calcification is rare. ${ }^{1,4}$ The radiological appearance correlates closely with the histological grade. High-grade tumours have indistinct and irregular margins while low-grade tumours have sharp 


\section{CASE REPORT}

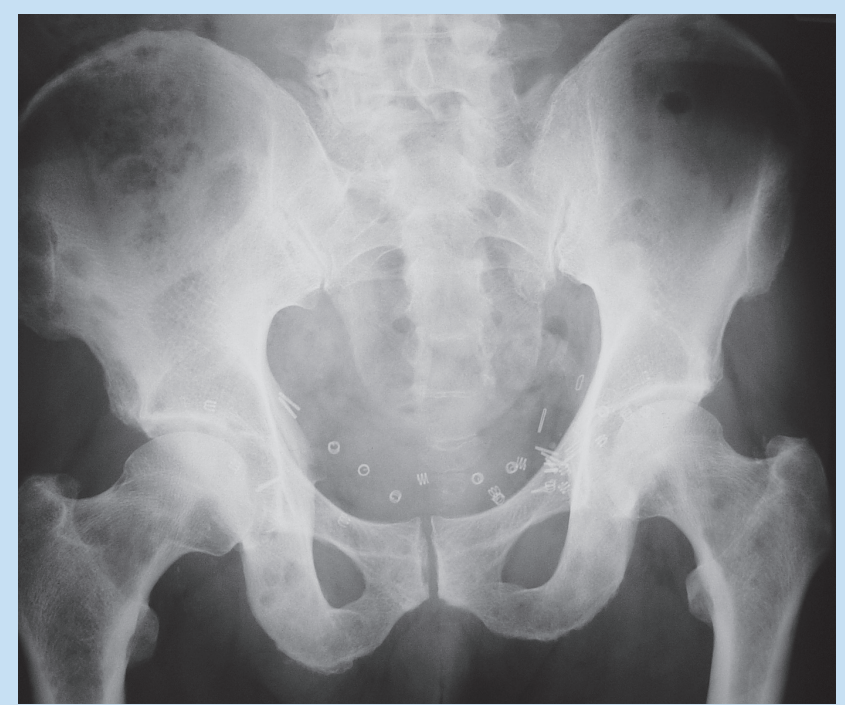

Fig. 1d.

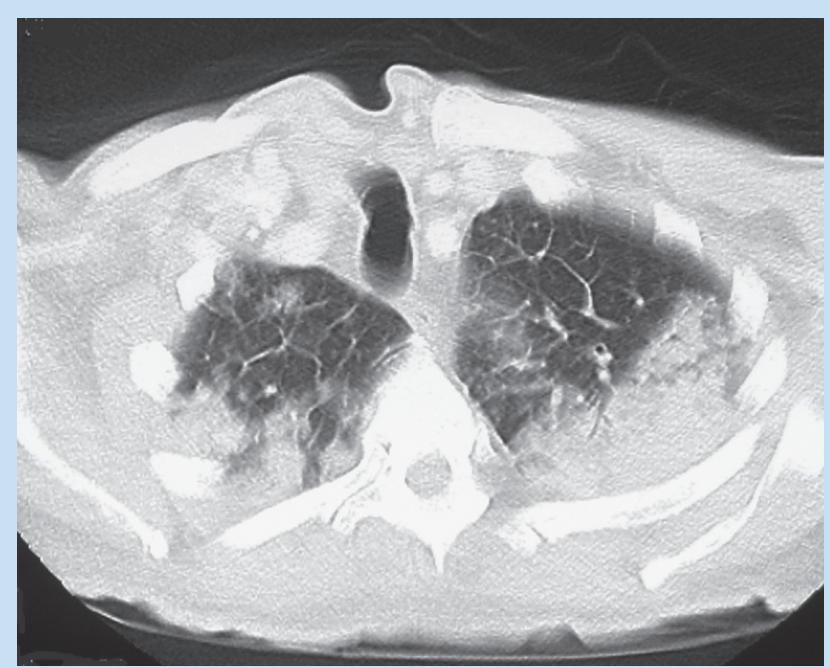

Fig. 2a. CT of the chest - lung windows. Multiple, fairly poorly circumscribed, subpleural nodules on a background of alveolar airspace disease and pleural effusions. This constitutes most probably metastatic disease on a background of infective changes within the lung parenchyma.

demarcated edges. ${ }^{1}$ Cortical destruction often occurs, but periosteal reaction is uncommon. ${ }^{2}$ It is essential to do a complete skeletal survey with plain films, MRI or scintigraphy. MRI can demonstrate occult lesions. There is intermediate signal intensity on T1W and high signal intensity on T2W images. Tumour enhances after IVI gadolinium injection. $^{2}$

CT can be used for assessment of cortical destruction and soft tissue penetration. ${ }^{2}$

Bone scintigraphy shows 'doughnut' lesions with increased uptake in the periphery and photopaenic zone in the central region of the tumour. $^{2}$

Preoperative angiography may show unseen lesions on CT and MRI. In some cases pre-operative tumour embolisation can be performed, which can reduce bleeding at the time of operation. ${ }^{2}$

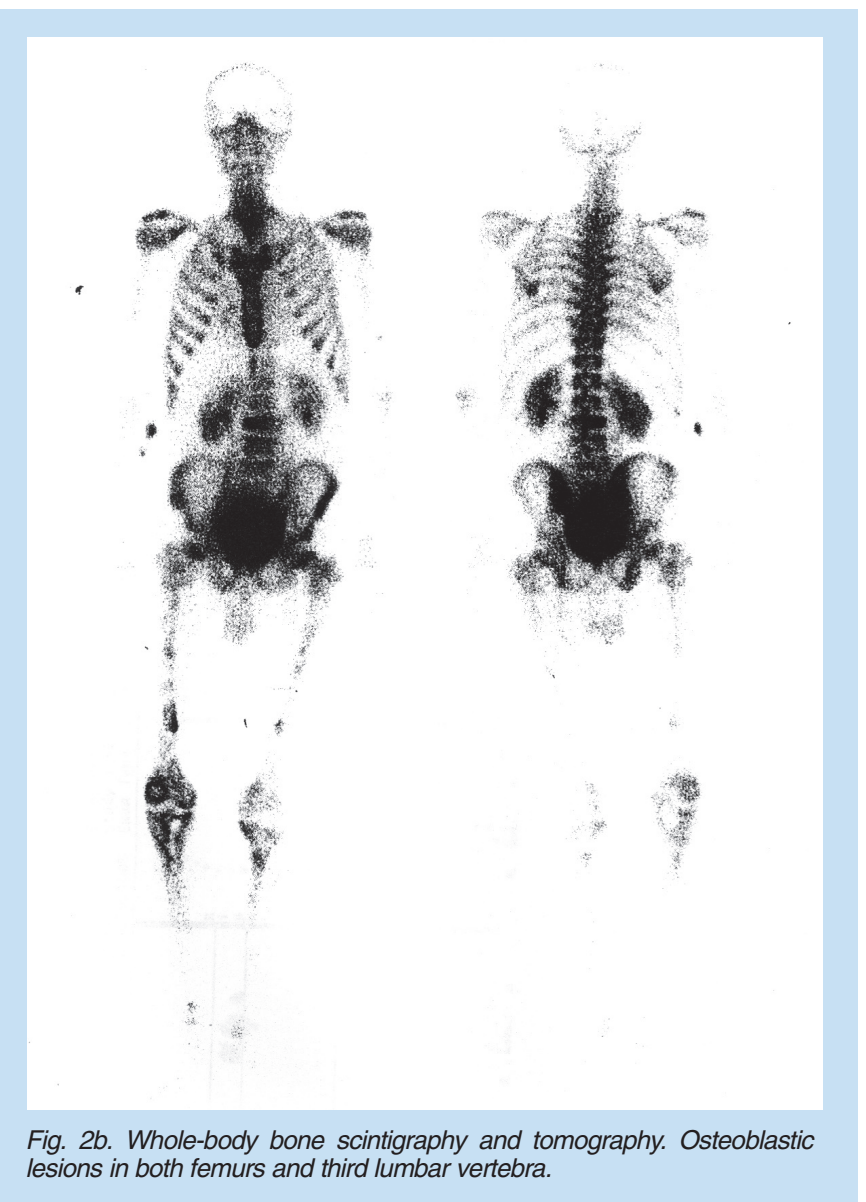

The radiological differential diagnosis includes epitheloid haemangioma, ${ }^{4}$ metastases, angiosarcoma, ${ }^{1,2}$ fibrous dysplasia ${ }^{2}$ and melanoma. ${ }^{1}$ Definitive diagnosis requires histopathological examination.

Treatment is determined by the grade, location and spread of the tumour. The ultimate goal remains that of cure. Wide resection of the tumour is recommended. ${ }^{1,2}$ Limb-saving procedures should be done when possible. Radiation therapy is indicated for a widespread or irresectable tumour in an attempt to stop local tumour progression. Radiotherapy is not recommended as an alternative to surgical treatment.

The role of chemotherapy has not been adequately assessed because of infrequent application. ${ }^{2}$

\section{Conclusion}

Keep this tumour in mind as a diagnostic possibility when multiple lucent bone lesions resembling metastases are seen on radiographs.

1. Cansiz H, Yener M, Dervisoglu S, Kalekoglu N. Hemangioendothelioma of the frontal bone in a child. Journal of Craniofacial Surgery 2003; 14: 724 - 728

2. Gosheger G, Hardes J, Ozaki T, Horst E, Burger H, Winkelmann W. The multicentric epitheloid hemangioendothelioma of bone: a case example and review of the literature. J Cancer Res Clin Oncol 2002; 128. $11-18$

3. Palsson B. Epitheloid hemangioendothelioma. Acta Oncologica 1999; 38: 659 - 661 .

4. Lewis VO, Montag AG, Simon MA. Epitheloid hemangiomas of bone. Clin Orthop 2003; 407: 167 - 172. 